\title{
Often Forgetting to do Things: What, Why and How not to?
}

\author{
David Shum \\ Griffith Health Institute, Griffith University, Gold Coast, Australia
}

\begin{abstract}
We rely on prospective memory (PM) to carry out intended actions at an appropriate time in the future. This ability is very important for day-to-day functioning, for example, remembering to take medication, attend appointments and pay bills. The literature covering the topic of PM has increased exponentially in the past 2030 years. This paper will describe this relatively novel form of memory and discuss the debilitating effect PM deficits have on everyday living. It will also examine the populations who experience significantly more frequent and severe PM impairments and explore the causes and mechanisms of PM impairments by reviewing behavioural, clinical, genetic and neuroimaging studies. The paper will conclude by discussing the latest research into ways of treating PM impairments in clinical populations. Where applicable, examples of research conducted by the author and his colleagues will be used to illustrate these topics.
\end{abstract}

Keywords: prospective memory, impairments, clinical samples, genetics, neuroimaging

\section{What is Prospective Memory?}

The ability to remember to do something planned in the future is referred to as prospective memory (PM). People use this kind of memory every day. We require it to remember to carry our keys when we go to work, or to make ourselves a cup of tea when the kettle has boiled. Failures in PM on these examples of day-to-day tasks often do not have drastic or negative consequences. An example of when they did was in 1999, when famous cellist Yo-Yo Ma left his \$2.5 million Montaganana cello in the trunk of a New York Taxi. There have also been cases where patients have sued their doctors for leaving surgical instruments inside their bodies after operations. It is evident that these isolated incidences can cause negative outcomes. It follows that people who experience PM impairments have ongoing difficulties in their everyday life and functioning. The concept of PM can be dated back to 1901 when Sigmund Freud described the following construct in his book The psychopathology of everyday life:

An intention is an impulse for an action which has already found approbation, but whose execution is postponed for a suitable occasion. Now, in the interval thus created sufficient change may take place in the motive to prevent the intention from coming to execution. It is not, however, forgotten, it is simply revised and omitted. (p. 92)

McDaniel and Einstein (2007) have described PM as remembering to carry out intended actions at an appropriate point in the future. Other characteristics of PM include: the intention cannot be carried out at the time of encoding, the delay between intention and action must be filled with an ongoing task, there is no explicit prompt or reminder by others and the ongoing task needs to be interrupted in order to carry out the intended task.

There are three types of PM. The first occurs when the intended action is linked to a specific time (e.g., when you wake up) or after a period of elapsed time (e.g., after 3 hours). This type of $\mathrm{PM}$ is described as time-based. Event-based is the second type of PM and refers to when the intended action is linked to a specific cue (e.g., posting a letter when one walks past a mailbox); or it may be linked to the end of a specific activity (e.g., taking medication after a meal), in which case it is the third type of PM - activity-based.

Address for correspondence: David Shum, Griffith Health Institute, Griffith University, Gold Coast, Australia. E-mail: d.shum@griffith.edu.au 


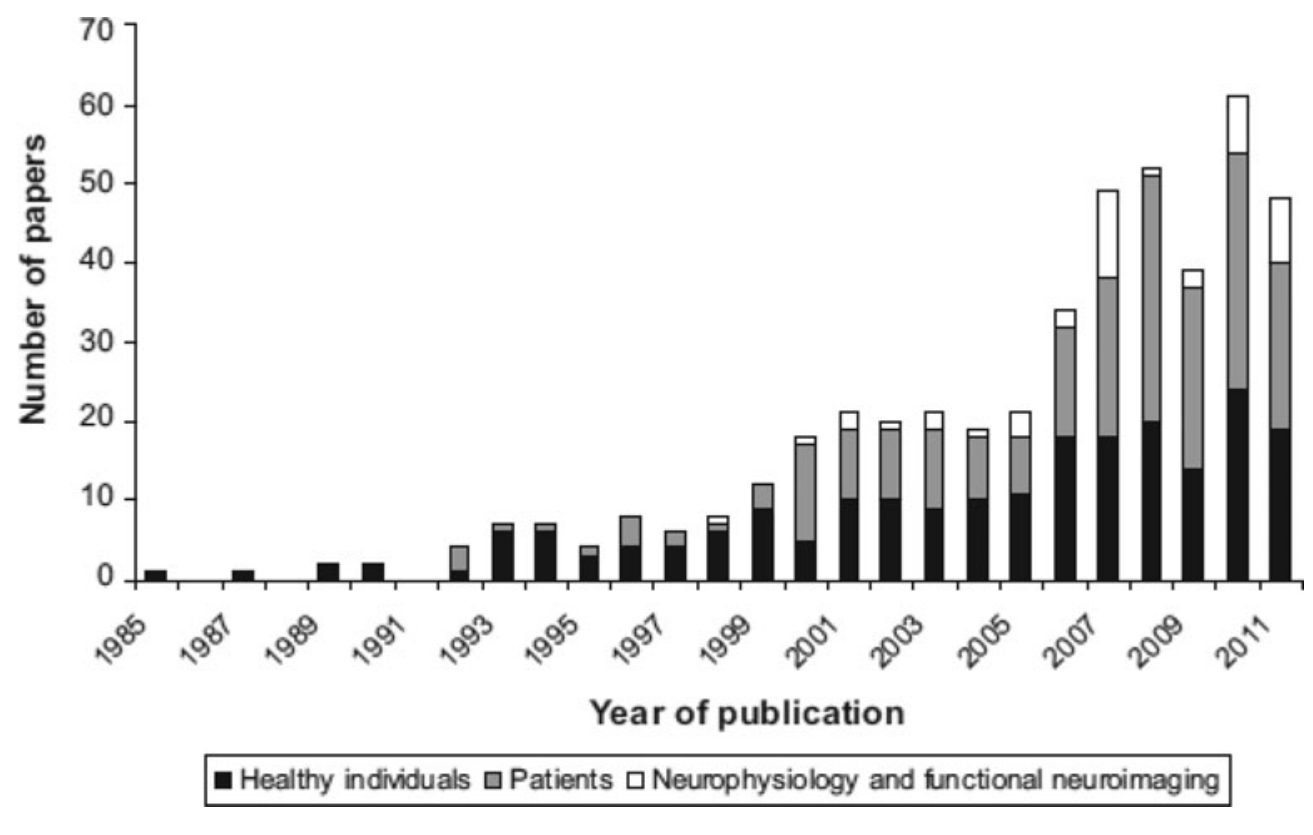

FIGURE 1

Number of papers exploring prospective memory in healthy individuals, patients and using neurophysiology and functional imaging methods. From Carlesimo and Costa (2011), reprinted with permission.

Ellis (1996) describes five phases of PM: (1) formation and encoding of intention and action; (2) retention interval; (3) performance interval; (4) initiation and execution of intended action; and (5) evaluation of outcome. These phases are used in research to identify in which component of the PM processs people experience deficits, whether this is different in different populations, and how interventions can be targeted to assist with these impairments.

As shown in Figure 1, the research exploring PM has grown exponentially in the past 20 30 years. Fifteen years ago PM was making such an influential impact on psychological and neurological literature that in 2000 the first International Conference on Prospective Memory was held at the University of Hertfordshire. Since then there have been two other international conferences, with the fourth conference to be held in Naples, Italy in May, 2014.

As noted, there is a difference between isolated PM lapses and PM impairments. Impairment is defined as a 'loss or abnormality of psychological, physiological, or anatomical structure or function' (World Health Organization, 1980, p. 48). Impairments can result from a lack of ability to perform activities in the manner or in the range consid- ered normal for a human being. Daniel Schacter (1996) describes the distinct difference in levels of concern about occasionally forgetting where one places his/her car keys or failing to come up with the name of a friend, and the more worrisome memory impairments such as forgetting that one possesses a car or not being able to clearly remember one's own name. PM has been identified as a critical element involved in the control and co-ordination of cognitive functions that are fundamental to our ability to complete a lot of real-world activities (Ellis \& Kvavilashvili, 2000). These PM failures have been shown to have a greater impact on the lives of carers than retrospective memory (RM) failures (Smith, Della Sala, Logie, \& Maylor, 2000) and have also shown to cause more stress and concern than RM failures (Ward, Shum, Dick, McKinlay, \& Baker-Tweeney, 2004).

\section{Clinical Groups with Prospective Memory Impairments and Causes of Prospective Memory Impairments}

Kliegel, Jägar, Altgassen and Shum (2008) have identified a growing list of clinical populations who experience PM impairments. These include people 


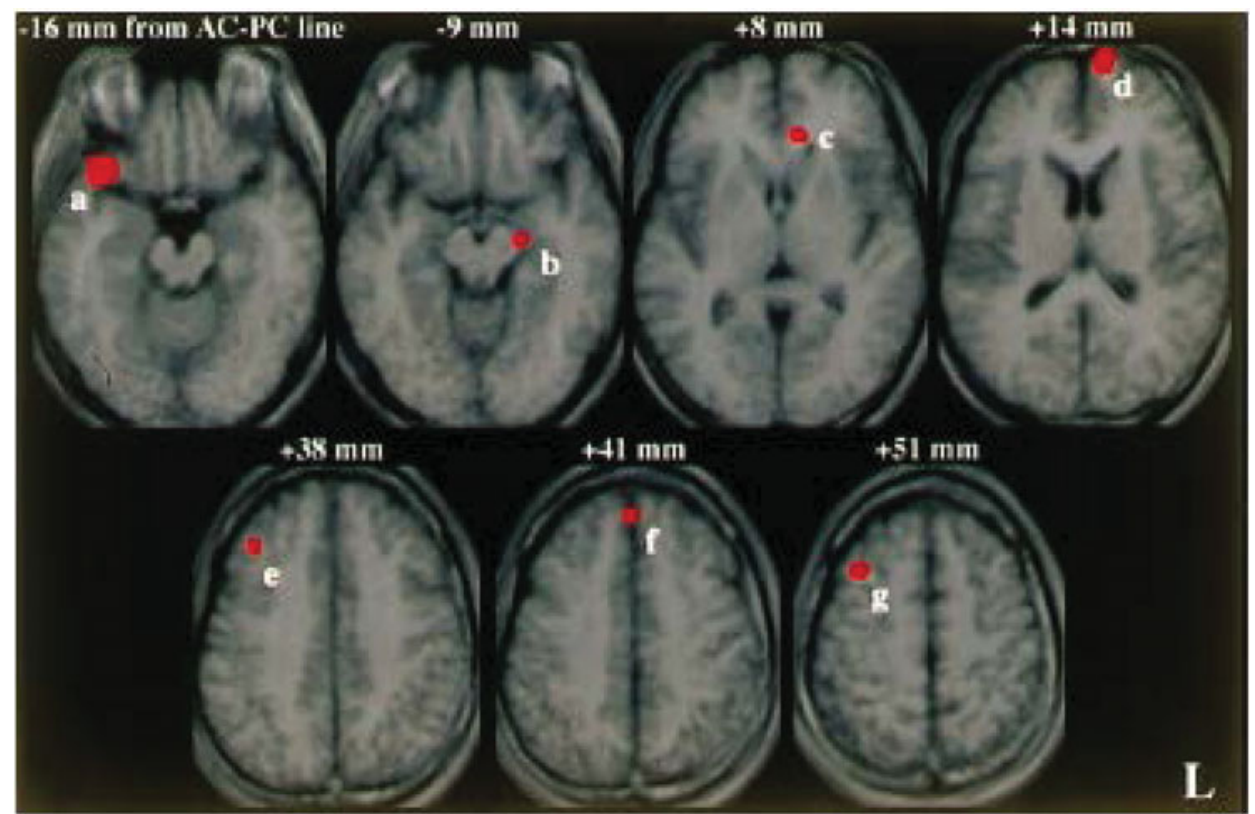

FIGURE 2

(Colour online) Brain areas activated during performance of prospective memory task. From Okuda et al. (1998), reprinted with permission.

living with dementia, Parkinson's disease, multiple sclerosis, human immunodeficiency virus, herpes simplex encephalitis, schizophrenia, affective disorders, attention deficit hyperactivity disorder, brain injury and people with substance abuse.

Following the identification and understanding of the negative impact of PM impairments on people's everyday functioning, researchers have since tried to elucidate the main causes of these deficits. There have been several lines of investigation, including neuroimaging, correlation with executive functions, manipulation of neurocogitive processes and genetics.

Neuroimaging techniques help by identifying the location(s) in the brain involved in PM behaviour and PM impairment. A study conducted by Okuda et al. (1998) recruited six right-handed males to perform a dual-task PM paradigm (Einstein \& McDaniel, 1990). As shown in Figure 2, this task activated the dorsolateral and ventrolateral prefrontal cortices, the left frontal pole and the anterior cingulate gyrus, left parahippocampal gyrus and midline medial frontal lobe. The authors concluded that these areas were involved in holding the intention, monitoring PM cue, and switching between ongoing and PM tasks.
Simons, Schölvinck, Gilbert, Frith and Burgess (2006) conducted a similar study using eight righthanded males and eight right-handed females and aimed to show a dissociation between cue identification and intention retrieval. Their results indicated a behavioural but not neural dissociation with activation in BA10 (lateral BA10 activation and medial BA10 deactivation), as shown in Figure 3. The researchers concluded that there is a common neural basis for PM cue identification and intention retrieval in area BA10.

A review of functional imaging studies of PM conducted by Burgess, Gonen-Yaacovi and Volle (2011) concludes that within the rostral prefrontal cortex there is a medial-lateral dissociation. While ongoing task performance activates the medial rostral prefrontal cortex, processes relating to PM seem to activate the lateral region of the area. In addition to the prefrontal area, the parietal lobe (e.g., areas BA40, BA7 and BA32) is also found to be activated during prospective remembering.

My colleagues and I have explored how PM correlates with executive functions, to try to highlight what may contribute to PM ability. Shum, Levin and Chan (2011) recently conducted a review of the literature of PM in patients with closed 


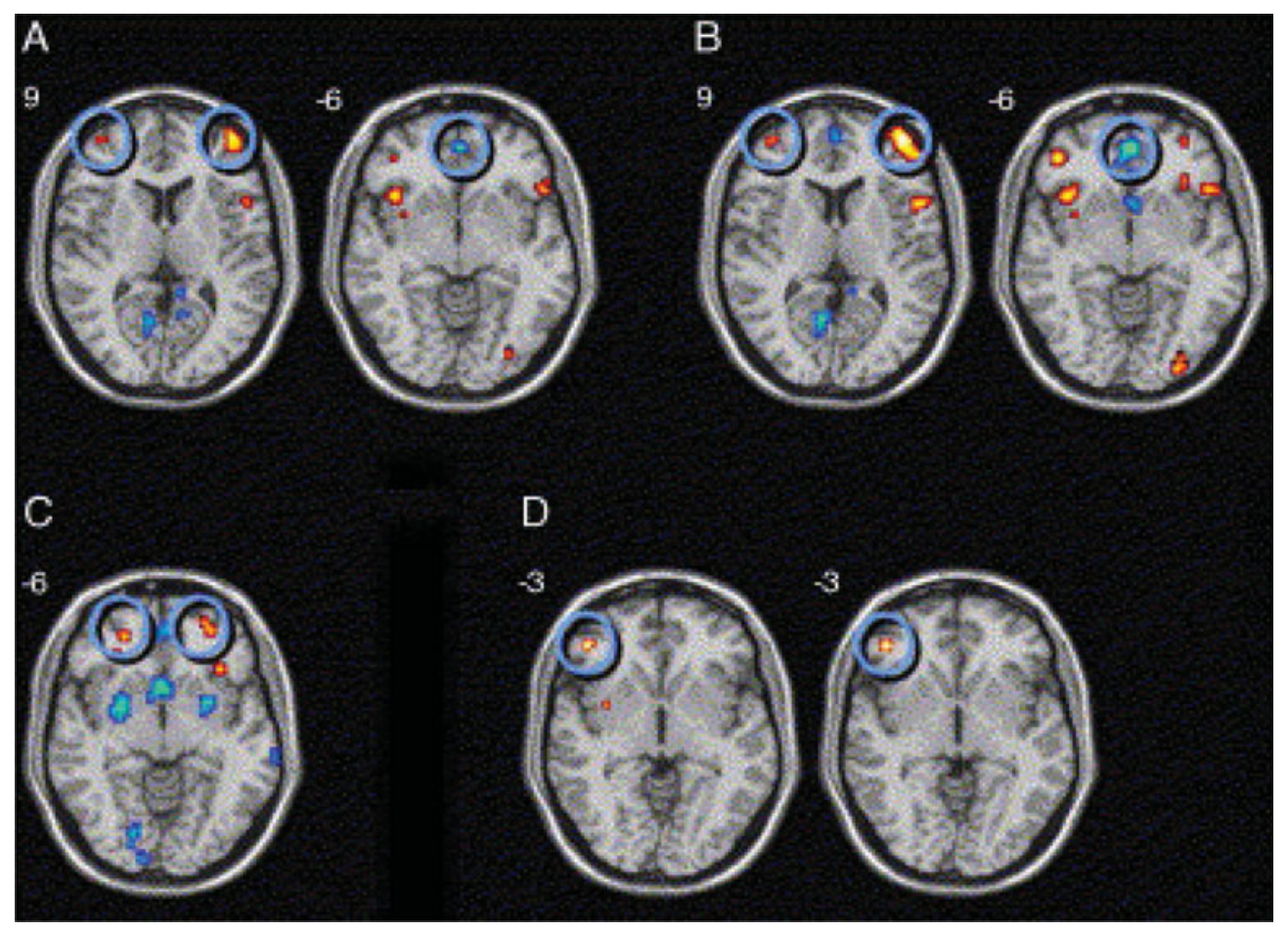

FIGURE 3

(Colour online) Brain areas involved in prospective memory. From Simons et al. (2006), reprinted with permission.

head injury and identified how their PM ability correlated with executive functions, as evidenced in Figure 4.

Another avenue of exploring the cause of PM deficits has been to manipulate neurocognitive processes in patients with schizophrenia (Shum, Ungvari, Tang, \& Leung, 2004). The research included 60 individuals with long-term schizophrenia and 60 controls. Three PM tasks (time-, event- and activity-based) were administered to the participants. Einstein and McDaniel's (1990) dual-task paradigm was implemented and results indicated a significant group $\times$ task interaction, suggesting that the prefrontal-lobe process of self-initiated retrieval is involved in PM. This finding supports the idea that manipulation of cognitive processes is useful for clarifying the causes of PM deficits. Furthermore, these findings have implications for the assessment and treatment of individuals with schizophrenia.

Similar research has been conducted with individuals with traumatic brain injury (TBI; Maujean, Shum, \& McQueen, 2003). The aim of this research was to establish the effect of cognitive demand on ongoing PM tasks. Participants included 14 people with severe TBI and 14 controls. The participants were required to undertake two tasks. A lexical-decision task which had two levels of cognitive demand (low and high) was used as an ongoing task, and an event-based PM task was used which involved performing a specific action whenever a target stimulus appeared during the ongoing task. Results showed significant main effects of both Group and Cognitive Demand and a significant two-way interaction, suggesting that working memory is important and involved in PM. Several neurocognitive tests were also administered to assess the relationship between prefrontal lobe functions and PM. These tests were the Letter-Number Sequencing Test, the Tower of London Test and the Controlled Oral Word Association Test. It was concluded that prefrontal-lobe function, which includes allocation and switching of cognitive resources, is involved in ongoing PM tasks. Furthermore, it was concluded that these findings help to clarify the nature of PM impairments experienced by people with TBI. 
Study name

Kinsella et al. 2009 EF

Knight et al. 2005 EF

Mathias \& Mansfield 2005 EF

Maujean \& Shum 2003 EF

Schimitter-Edgecombe \& Wright 2004 EF
Correlation and $95 \% \mathrm{Cl}$

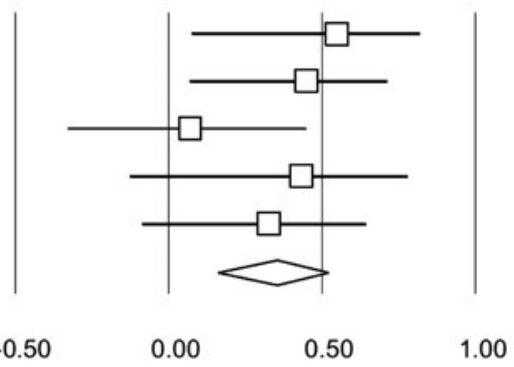

\section{FIGURE 4}

Results of meta-analysis for relationship between PM and executive functions in patients with closed head injury. From Shum et al. (2011), reprinted with permission.

Recent research has identified genetic markers linked to PM and can therefore help identify the cause of PM impairments. A study conducted by the author and colleagues (Dongés et al., 2012) targeted a known association between certain genes and PM. The genes used were the APOE $\varepsilon 4$ allele (a known risk factor for Alzheimer's disease) and COMT Val 158 polymorphism (implicated in schizophrenia). A battery of self-report measures were used to test 197 healthy adults (131 female and 66 male) aged between 16 and 51 years old. One of the PM tools used was the Comprehensive Assessment of Prospective Memory (CAPM) (Shum \& Fleming, 2014). Results indicated that individuals with the APOE $\varepsilon 4$ allele reported significantly more PM problems than those with other alleles.

\section{Can Prospective Memory Impairments be Treated?}

Thus far, this paper has described the construct of PM, explained its importance in everyday functioning, identified clinical populations who experience prospective memory impairments and explored the possible causes of PM impairments. Given the substantial impact these impairments have on people's lives, some PM researchers have conducted studies to find out whether or not impairments can be treated.

In 2011 the author and colleagues published a randomized controlled trial (RCT) of PM rehabilitation in 45 adults with TBI (Shum, Fleming,
Gill, Gullo, \& Strong, 2011). The RCT examined the efficacy of compensatory PM training, preceded by self-awareness training, for adult patients with moderate to severe TBI. Results indicated the compensatory PM training led to improvement on scores of PM, as well as strategy use. The effects of self-awareness training were not as clear. This study provides preliminary support for a relatively short-duration and low-intensity compensatory approach for treatment of PM impairments in individuals with TBI.

Support has also been found for the utilization of a technique called implementation intentions to improve PM performance (Khoyratty et al., 2014). The author and colleagues tested 30 early psychosis patients and 33 healthy controls using a $2 \times$ 2 design (instruction $\times$ group). Participants were either instructed to use implementation intentions or were just given the standard instructions. The findings revealed that individuals with early psychosis had PM deficits and that these individuals benefited from implementation intentions. Furthermore, these findings suggested that this technique may serve as a simple and cost-effective technique for improving PM.

\section{Future Directions}

PM research would benefit considerably from the development of better, more ecologically valid PM tasks. Developers must also endeavour to focus on and assess independent indices for various PM components. Benefits to this research area would 
also come through a better understanding of the association between PM task performance and outcome measures. The benefits of advancing technology have already been outlined in the section of this paper on neuroimaging; however, it should be highlighted that more can be learnt about the nature of PM through staying up to date with the latest technologies. Finally, and most importantly, to ensure that the people experiencing these debilitating deficits benefit from research, there must be a deliberate focus on researching rehabilitation. It would be extremely beneficial if research in this area could establish a generalised assessment and training program to maintain gains made through intervention strategies.

\section{Conclusion}

This paper has provided an overview of the development of a novel memory construct. It has highlighted the impact that deficits in this kind of memory have on people's everyday lives and explained the latest research into what might cause or be associated with PM ability. Interventions that have shown some success in the improvement of $\mathrm{PM}$ deficits and ideas for future research in this area were then described.

\section{References}

Burgess, P.W., Gonen-Yaacovi, G., \& Volle, E. (2011). Functional neuroimaging studies of prospective memory: What have we learnt so far? Neuropsychologia, 49(8), 2246-2257.

Carlesimo, G.A., \& Costa, A. (2011). An introduction to the special issue on the neuropsychology of prospective memory. Neuropsychologia, 49(8), 2143-2146.

Dongés, B., Haupt, L.M., Lea, R.A., Chan, R.C.K., Shum, D.H.K., \& Griffiths, L.R. (2012). Role of the apolipoprotein E and catechol-O-methyltransferase genes in prospective and retrospective memory traits. Gene, 506(1), 135-140.

Einstein, G.O., \& McDaniel, M.A. (1990). Normal aging and prospective memory. Journal of Experimental Psychology: Learning, Memory and Cognition, 16(4), 717-726.

Ellis, J. (1996). Prospective memory or the realization of delayed intentions: A conceptual framework for research. In M. Brandimonte, G.O. Einstein \& M.A. McDaniel (Eds.), Prospective memory: Theory and applications (pp. 1-22). Mahwah, NJ, US: Lawrence Erlbaum Associates.

Ellis, J., \& Kvavilashvili, L. (2000). Prospective memory in 2000: Past, present, and future directions. Applied Cognitive Psychology, 14(7), S1-S9.

Freud, S. (1901). The psychopathology of everyday life. New York: W.W. Norton.
Khoyratty, N., Wang, Y., O'Gorman, J., Lloyd, C., Williams, P.L., Chan, R.C.K., \& Shum, D.H.K. (2014). Forming implementation intentions improves prospective memory in early psychosis (manuscript submitted for publication).

Kliegel, M., Jäger, T., Altgassen, M., \& Shum, D. (2008). Clinical neuropsychology of prospective memory. In M. Kliegel, M.A. McDaniel \& G.O. Einstein (Eds.), Prospective memory: Cognitive, neuroscience, developmental, and applied perspectives (pp. 283-308). New York, NY: Taylor \& Francis Group/Lawrence Erlbaum Associates.

Maujean, A., Shum, D., \& McQueen, R. (2003). Effect of cognitive demand on prospective memory in individuals with traumatic brain injury. Brain Impairment, $4(2), 135-145$.

McDaniel, M.A., \& Einstein, G.O. (2007). Prospective memory: An overview and synthesis of an emerging field. California: Sage Publications.

Okuda, J., Fujii, T., Yamadori, A., Kawashima, R., Tsukiura, T., Fukatsu, R., . . . Fukuda, H. (1998). Participation of the prefrontal cortices in prospective memory: evidence from a PET study in humans. Neuroscience Letters, 253(2), 127-130.

Schacter, D. (1996). Searching for memory: The brain, the mind, and the past. New York: Basic Books.

Shum, D.H.K., \& Fleming, J., (2014). Comprehensive assessment of prospective memory: Clinical assessment and user manual. Gold Coast: Griffith Health Institute.

Shum, D., Fleming, J., Gill, H., Gullo, M.J., \& Strong, J. (2011). A randomized controlled trial of prospective memory rehabilitation in adults with traumatic brain injury. Journal of Rehabilitation Medicine, 43(3), 216-223.

Shum, D., Levin, H., \& Chan, R.C.K. (2011). Prospective memory in patients with closed head injury: A review. Neuropsychologia, 49(8), 2156-2165.

Shum, D., Ungvari, G.S., Tang, W.-K., \& Leung, J.P. (2004). Performance of schizophrenia patients on time-, event-, and activity-based prospective memory tasks. Schizophrenia Bulletin, 30(4), 693-702.

Simons, J.S., Schölvinck, M.L., Gilbert, S.J., Frith, C.D., \& Burgess, P.W. (2006). Differential components of prospective memory? Evidence from fMRI. Neuropsychologia, 44(8), 1388-1397.

Smith, G., Della Sala, S., Logie, R.H., \& Maylor, E.A. (2000). Prospective and retrospective memory in normal ageing and dementia: A questionnaire study. Memory, 8(5), 311-321.

Ward, H., Shum,, D., Dick, B., McKinlay, L., \& BakerTweeney, S. (2004). Interview study of the effects of paediatric traumatic brain injury on memory. Brain Injury, 18, 471-495.

World Health Organization. (1980). International classification of impairments, disabilities, and handicaps: A manual of classification relating to the consequences of disease. Geneva: WHO. 